Louisiana State University

LSU Digital Commons

$11-25-2005$

\title{
Insights from the x-ray crystal structure of coral 8R-lipoxygenase: Calcium activation via a C2-like domain and a structural basis of product chirality
}

Michael L. Oldham

Louisiana State University

Alan R. Brash

Vanderbilt University School of Medicine

Marcia E. Newcomer

Louisiana State University

Follow this and additional works at: https://digitalcommons.Isu.edu/biosci_pubs

\section{Recommended Citation}

Oldham, M., Brash, A., \& Newcomer, M. (2005). Insights from the x-ray crystal structure of coral 8Rlipoxygenase: Calcium activation via a C2-like domain and a structural basis of product chirality. Journal of Biological Chemistry, 280 (47), 39545-39552. https://doi.org/10.1074/jbc.M506675200 


\title{
Insights from the X-ray Crystal Structure of Coral $8 R$-Lipoxygenase \\ CALCIUM ACTIVATION VIA A C2-LIKE DOMAIN AND A STRUCTURAL BASIS OF PRODUCT CHIRALITY*
}

Received for publication, June 20, 2005, and in revised form, September 7, 2005 Published, JBC Papers in Press, September 14, 2005, DOI 10.1074/jbc.M506675200

\author{
Michael L. Oldham ${ }^{\ddagger \S}$, Alan R. Brash", and Marcia E. Newcomer ${ }^{\ddagger 1}$ \\ From the ${ }^{\ddagger}$ Department of Biological Sciences, Louisiana State University, Baton Rouge, Louisiana 70803 and the Departments of \\ ${ }^{\S}$ Biochemistry and "Pharmacology, Vanderbilt University School of Medicine, Nashville, Tennessee 37232
}

\begin{abstract}
Lipoxygenases (LOXs) catalyze the regio- and stereospecific dioxygenation of polyunsaturated membrane-embedded fatty acids. We report here the $3.2 \AA$ resolution structure of $8 R$-LOX from the Caribbean sea whip coral Plexaura homomalla, a LOX isozyme with calcium dependence and the uncommon $R$ chiral stereospecificity. Structural and spectroscopic analyses demonstrated calcium binding in a C2-like membrane-binding domain, illuminating the function of similar amino acids in calcium-activated mammalian 5-LOX, the key enzyme in the pathway to the pro-inflammatory leukotrienes. Mutation of $\mathrm{Ca}^{2+}$-ligating amino acids in $8 R$ - LOX resulted not only in a diminished capacity to bind membranes, as monitored by fluorescence resonance energy transfer, but also in an associated loss of $\mathrm{Ca}^{2+}$-regulated enzyme activity. Moreover, a structural basis for $R$ chiral specificity is also revealed; creation of a small oxygen pocket next to $\mathrm{Gly}^{428}$ (Ala in all $S$-LOX isozymes) promoted C-8 oxygenation with $R$ chirality on the activated fatty acid substrate.
\end{abstract}

Lipoxygenases (LOXs) ${ }^{2}$ are non-heme iron dioxygenases that catalyze the regio- and stereospecific formation of fatty acid hydroperoxides from polyunsaturated fatty acids $(1,2)$. The substrates for the plant enzymes are generally linoleic and linolenic acids, whereas the animal enzymes catalyze the peroxidation of arachidonic acid in the biosynthesis of leukotrienes and hydroxy fatty acids. Both plant and animal LOXs are composed of two domains: a smaller N-terminal $\beta$-barrel domain and a larger $\alpha$-helical catalytic domain. The fold of the $\beta$-barrel domain resembles that of a "C2" module, calcium-dependent membrane-targeting domains found in many protein kinases and phospholipases (3), and is equivalent in topology to the C2-like membrane-binding domain of gangrene $\alpha$-toxin (4). Similarly, the $\beta$-barrel domains of LOXs have also been shown to mediate membrane binding (5-7).

The larger $\alpha$-helical catalytic domain $(\sim 540$ amino acids in rabbit 15S-LOX1) contains the non-heme iron essential for activity positioned

\footnotetext{
* This work was supported in part by National Institutes of Health Grants GM 55420 (to M. E. N.) and GM 53638 (to A. R. B.) and by the Louisiana Governor's Biotechnology Initiative. The costs of publication of this article were defrayed in part by the payment of page charges. This article must therefore be hereby marked "advertisement" in accordance with 18 U.S.C. Section 1734 solely to indicate this fact.

The atomic coordinates and structure factors (code 1zq4) have been deposited in the Protein Data Bank, Research Collaboratory for Structural Bioinformatics, Rutgers University, New Brunswick, NJ (http://www.rcsb.org/).

${ }^{1}$ To whom correspondence should be addressed: Dept. of Biological Sciences, 202 Life Sciences Bldg., Louisiana State University, Baton Rouge, LA 70803. Tel.: 225-578-7383; Fax: 225-578-7258; E-mail: newcomer@Isu.edu.

2 The abbreviations used are: LOX, lipoxygenase; FRET, fluorescence resonance energy transfer; dansyl-DHPE， N-(5-dimethylaminonaphthalene-1-sulfonyl)-1,2-dihexadecanoyl-sn-glycero-3-phosphoethanolamine; r.m.s.d., root mean square deviation.
}

midway in a deep cavity that accommodates the hydrocarbon portion of the substrate (8). In mammalian 15S-LOX1, an exposed Arg is proposed to tether the carboxylate of the fatty acid substrate at the surface. Regioand stereospecificities of the various isozymes are thought to be governed by the shape and depth of the pocket, which must both position the substrate for attack and allow the approach of $\mathrm{O}_{2}$, as well as the orientation (head versus tail first) of the substrate in the cavity $(9,10)$. Although the overall fold and geometry of the non-heme iron-binding site are conserved with respect to the plant and animal enzymes, there are significant differences in size, sequence, and specificity determinants between these LOX classes (11).

The structures of rabbit 15S-LOX1 and the plant LOXs L1 and L3 have been described $(8,13-15)$, but no structure of a LOX that catalyzes dioxygenation with $R$ stereochemistry has been reported. Recently, Coffa and Brash (16) observed that product stereochemistry is strongly influenced by a single amino acid: an Ala in the binding site of $S$-LOXs is a Gly in the corresponding position of R-LOXs. Single-site mutant LOXs in which Ala is exchanged with Gly and vice versa yield products opposite in stereochemistry to those of the parent wild-type proteins.

Oxylipin biosynthesis studies have led to the identification of an $8 R$-LOX as part of a fusion protein with an allene-oxide synthase in the soft coral Plexaura homomalla (17). The C-terminal LOX domain shares significant sequence identity (41\%) with the $\mathrm{Ca}^{2+}$-regulated mammalian 5-LOX, an asthma drug target due to its pivotal role in the biosynthesis of the potent bronchoconstrictor leukotrienes. In contrast to mammalian 5-LOX (18), 8R-LOX is remarkably stable and thus amenable to crystallographic studies. An important aim of our structural analysis of $8 R$-LOX is to explore the mechanistic basis of stereo control in LOXs. We report here the 3.2- $\AA$ resolution structure of $\mathrm{Ca}^{2+}$-activated $8 R$-LOX and demonstrate its calcium-dependent membrane targeting with fluorescence resonance energy transfer (FRET).

\section{MATERIALS AND METHODS}

Site-directed Mutagenesis-Construction of mutants was performed using the QuikChange site-directed mutagenesis kit (Stratagene). The desired mutations were confirmed by sequencing.

Expression and Purification-Recombinant 8R-LOX and associated mutants were expressed with an $\mathrm{N}$-terminal $\mathrm{His}_{6}$ tag in BL21(DE3) cells (Novagen). Following isopropyl $\beta$-D-thiogalactopyranoside induction at $A_{600 \mathrm{~nm}}=0.7$, cells were grown in $2 \times$ Yeast Tryptone medium for $24 \mathrm{~h}$ at $20^{\circ} \mathrm{C}$. Cells were harvested $\left(5000 \times g, 20 \mathrm{~min}, 4^{\circ} \mathrm{C}\right)$, frozen at $-80^{\circ} \mathrm{C}$, and subsequently lysed with BugBuster protein extraction reagent (Novagen) with DNase I added. Cell debris was removed by centrifugation at $46,000 \times g$ for $20 \mathrm{~min}$ at $4{ }^{\circ} \mathrm{C}$, and the supernatant was applied to nickel-nitrilotriacetic acid-agarose (Qiagen Inc.) equilibrated 
with $20 \mathrm{~mm}$ imidazole and $500 \mathrm{~mm} \mathrm{NaCl}$. After elution with $200 \mathrm{~mm}$ imidazole, the protein was further purified by ion-exchange chromatography using DE52 (Whatman) and Mono Q (Amersham Biosciences) resins to yield the purified product. Calcium was removed by dialysis of the concentrated protein samples in the presence of neutralized Chelex 100 (Bio-Rad) prior to dropwise flash-freezing in liquid $\mathrm{N}_{2}$ and storage at $-80^{\circ} \mathrm{C}$.

Crystallization-Plate-like crystals were grown in hanging-drop vapor diffusion plates in 6.25\% polyethylene glycol 8000 (Fluka), $100 \mathrm{~mm}$ imidazole acetate ( $\mathrm{pH} 8.0), 100 \mathrm{mM} \mathrm{CaCl}_{2}$, and $5 \%$ sucrose at $22{ }^{\circ} \mathrm{C}$. The crystals belong to space group P2 $1(a=69.6, b=78.0$, and $c=176.5 \AA$ and $\beta=96.7^{\circ}$ ) and diffract to $\sim 3.2 \AA$. There are two molecules/asymmetric unit.

Data Collection and Phasing-Data were collected at $100 \mathrm{~K}$ on a Nonius FR591 rotating anode fitted with a Mar345 detector. Crystals were flash-frozen after transfer to mother liquor solutions in which the concentration of ethylene glycol was incrementally increased to $40 \%$. Data processing and scaling were performed with Denzo and Scalepack (19). The structure of $8 R$-LOX was solved by molecular replacement with MOLREP (CCP4) (20) with a polyserine model of rabbit 15S-LOX1 (with which it shares $30 \%$ sequence identity) as the search model. Two molecules were positioned to give a correlation coefficient of 36.9 and an $R$-factor of 53.1. The first map was calculated with a polyalanine model based on the molecular replacement results and $2 F_{o}-F_{c}$ amplitudes and revealed clear electron density for the non-heme iron and chelating side chains. After multiple cycles of map interpretation with program $O(21)$ and refinement with strict non-crystallographic symmetry restraints in CNS (22), the final model has $R_{\text {work }}$ and $R_{\text {free }}$ values of 25.5 and 29.9, respectively.

Liposome Preparation-Large unilamellar vesicles were prepared from a mixture of $75 \mathrm{~mol}$ of phosphatidylcholine and $25 \mathrm{~mol}$ of phosphatidylserine with 11 passes in a mini-extruder assembly (Avanti Polar Lipids). Liposomes used in FRET studies also contained $N$-(5-dimethylaminonaphthalene-1-sulfonyl)-1,2-dihexadecanoyl-sn-glycero-3phosphoethanolamine (dansyl-DHPE; triethylammonium salt; Molecular Probes) at $5 \%(\mathrm{w} / \mathrm{w})$.

FRET Studies of $\mathrm{Ca}^{2+}$-regulated Tryptophan Membrane InsertionThe affinity of $8 R$-LOX for large unilamellar vesicles was determined by monitoring the decrease in Trp fluorescence upon the addition of $\mathrm{Ca}^{2+}$ to solutions of large unilamellar vesicles in which dansyl-DHPE was incorporated at $5 \%(\mathrm{w} / \mathrm{w})$. The concentration of the enzyme was $0.4 \mu \mathrm{M}$, and the liposome concentration varied from 10 to $80 \mu \mathrm{M}$ in a sample size of $3.4 \mathrm{ml}$. Fresh protein/dansyl-DHPE liposome solutions were prepared for each data point; emission spectra were scanned (excitation wavelength of $295 \mathrm{~nm}$ and emission wavelength of 320-400 nm) after the addition of $2 \mathrm{mM}$ EDTA; and the spectra were rescanned after the addition of $4 \mathrm{mM} \mathrm{CaCl}_{2}$. A plot of the fractional loss of Trp fluorescence signal $\left(\Delta F_{ \pm \text {calcium }} / F_{- \text {calcium }}\right)$ versus liposome concentration yielded a hyperbolic binding curve when fitted by nonlinear regression analysis with SigmaPlot.

Membrane insertion of wild-type and mutant $8 R$-LOXs was monitored by a loss in Trp fluorescence (excitation wavelength of $295 \mathrm{~nm}$ ) and a gain in dansyl fluorescence upon the addition of $\mathrm{Ca}^{2+}$ to solutions of $8 R$-LOX and large unilamellar vesicles in which dansyl-DHPE was incorporated at $5 \%(\mathrm{w} / \mathrm{w})$. LOX and phospholipid concentrations were 1.2 and $260 \mu \mathrm{M}$, respectively (in $50 \mathrm{~mm}$ Tris- $\mathrm{HCl}$ (pH 7.5) and $500 \mathrm{~mm}$ $\mathrm{NaCl}$ ), in a sample size of $185 \mu$ l. Initial fluorescence spectra were taken in the presence of $4 \mathrm{~mm} \mathrm{EDTA} ; \mathrm{CaCl}_{2}$ was added to a final concentration of $6 \mathrm{~mm}$ prior to the second trace. All emission spectra were recorded on a Jasco FP-6300 spectrofluorometer with the bandwidth and band pass
TABLE ONE

Data collection and refinement statistics

\begin{tabular}{|c|c|}
\hline \multicolumn{2}{|l|}{ Data collection } \\
\hline Space group & $\mathrm{P} 2_{1}$ \\
\hline \multicolumn{2}{|l|}{ Cell dimensions } \\
\hline$a, b, c(\AA)$ & $69.6,78.0,176.5$ \\
\hline$\alpha, \beta, \gamma$ & $90.0^{\circ}, 96.7^{\circ}, 90.0^{\circ}$ \\
\hline Resolution $(\AA)$ & 29.9 to 3.2 \\
\hline$R_{\text {merge }}^{a, b}$ & $10.1(38.4)$ \\
\hline$I / \sigma I$ & $9.7(2.3)$ \\
\hline Completeness (\%) & $98.5(82.3)$ \\
\hline Redundancy & $3.3(2.8)$ \\
\hline \multicolumn{2}{|l|}{ Refinement } \\
\hline Resolution $(\AA)$ & 29.9 to 3.2 \\
\hline No. reflections & 28,928 \\
\hline$R_{\text {work }} / R_{\text {free }}{ }^{c}$ & $25.5 / 29.9$ \\
\hline \multicolumn{2}{|l|}{ No. atoms } \\
\hline Protein & 10,436 \\
\hline Ligand/ion & 8 \\
\hline \multicolumn{2}{|l|}{$B$-Factors } \\
\hline Protein & 42.8 \\
\hline Ligand/ion & 41.1 \\
\hline \multicolumn{2}{|l|}{ r.m.s.d. } \\
\hline Bond length $(\AA)$ & 0.008 \\
\hline Bond angle & $1.51^{\circ}$ \\
\hline \multicolumn{2}{|c|}{$\begin{array}{l}{ }^{a} \text { Values in parentheses are for the highest resolution shell. } \\
{ }^{b} R_{\text {merge }}=\Sigma\left|I_{i}-\langle I\rangle\right| / \Sigma I_{i} \text {, where } I_{i} \text { is the intensity of the } i \text { th observation and }\langle I\rangle \text { is the } \\
\text { mean intensity of the reflection. } \\
{ }^{c} R=\Sigma|| F_{o}|-| F_{c} \| / \Sigma\left|F_{o}\right| \text {, where } F_{o} \text { and } F_{c} \text { are the observed and calculated structure } \\
\text { factor amplitudes, respectively. } R_{\text {free }} \text { was calculated using } 5 \% \text { of the total } \\
\text { reflections. }\end{array}$} \\
\hline
\end{tabular}

both set to $5 \mathrm{~nm}$. The data in Fig. 5 are reported as the normalized difference in fluorescence at $517 \mathrm{~nm}$ in the absence and presence of $\mathrm{CaCl}_{2}$ relative to that for the wild-type enzyme. Experiments were performed in triplicate and are reported as the mean \pm S.D. of three independent observations.

Stopped-flow Data Acquisition and Analysis-The effect of $\mathrm{Ca}^{2+}$ on 8R-LOX catalysis was examined in an Applied Photophysics Model SX17MV stopped-flow spectrometer operating in the absorbance mode at $25^{\circ} \mathrm{C}$. Product ( $8 R$-hydroperoxyeicosatetraenoic acid) formation was monitored at $235 \mathrm{~nm}$ with a cell path length of $0.2 \mathrm{~cm}$. The enzyme concentration was $10 \mathrm{~nm}$ in $50 \mathrm{~mm}$ Tris- $\mathrm{HCl}$ (pH 7.5), $500 \mathrm{~mm} \mathrm{NaCl}$, and $2 \mathrm{mM}$ EDTA. Assays were performed with $25 \mu \mathrm{M}$ arachidonic acid (Cayman Chemical) in the absence and presence of large unilamellar vesicles (260 $\mu \mathrm{M}$ phospholipid) and in the presence of $4 \mathrm{mM} \mathrm{CaCl}_{2}$ and large unilamellar vesicles. The reported concentrations of reagents are those in the observation cell. Each kinetic trace was the average of at least three individual measurements.

\section{RESULTS}

The Structure of $8 R-L O X$-The structure of $8 R$-LOX in the presence of calcium was solved to $3.2 \AA$ and refined to $R_{\text {work }}$ and $R_{\text {free }}$ values of 25.5 and 29.9, respectively (TABLE ONE). The overall fold is similar to that of rabbit 15S-LOX1 (root mean square deviation (r.m.s.d.) of $1.54 \AA$ for 576 of $662 \mathrm{C}-\alpha$ atoms), yet significant differences are found in the loops on one side of the $\beta$-barrel, in accessibility to the active site, and in the substrate-binding pocket of the catalytic domain. $8 R$-LOX encompasses amino acids 374-1066 of the natural fusion protein with alleneoxide synthase; but to simplify the discussion with respect to LOXs in general, we have renumbered $8 R$-LOX to amino acids 2-694. 
FIGURE 1. The structure of 8 R-LOX. $a$, the overall fold of $8 R$-LOX. Shown are the $\mathrm{N}$-terminal $\beta$-barrel (blue) and the $\alpha$-helical catalytic domain (red) with associated $\mathrm{Ca}^{2+}$ (yellow) and $\mathrm{Fe}^{3+}$ (magenta) ions and chelating residues. $b$, the $8 R$-LOX active site. Ligands $\mathrm{His}^{385}, \mathrm{His}^{390}, \mathrm{His}^{571}, \mathrm{Asn}^{575}$, and $\mathrm{Il}^{694}$ (yellow carbons) to the ferric iron are shown in a $2 F_{o}-$ $F_{c}$ electron density map contoured at $1.75 \sigma$. The substrate pocket-constricting $\mathrm{Leu}^{386}$ and Leu ${ }^{628}$ (magenta carbons) and $R$ stereochemistry determining $\mathrm{Gly}^{428}$ (green carbons) are also shown.
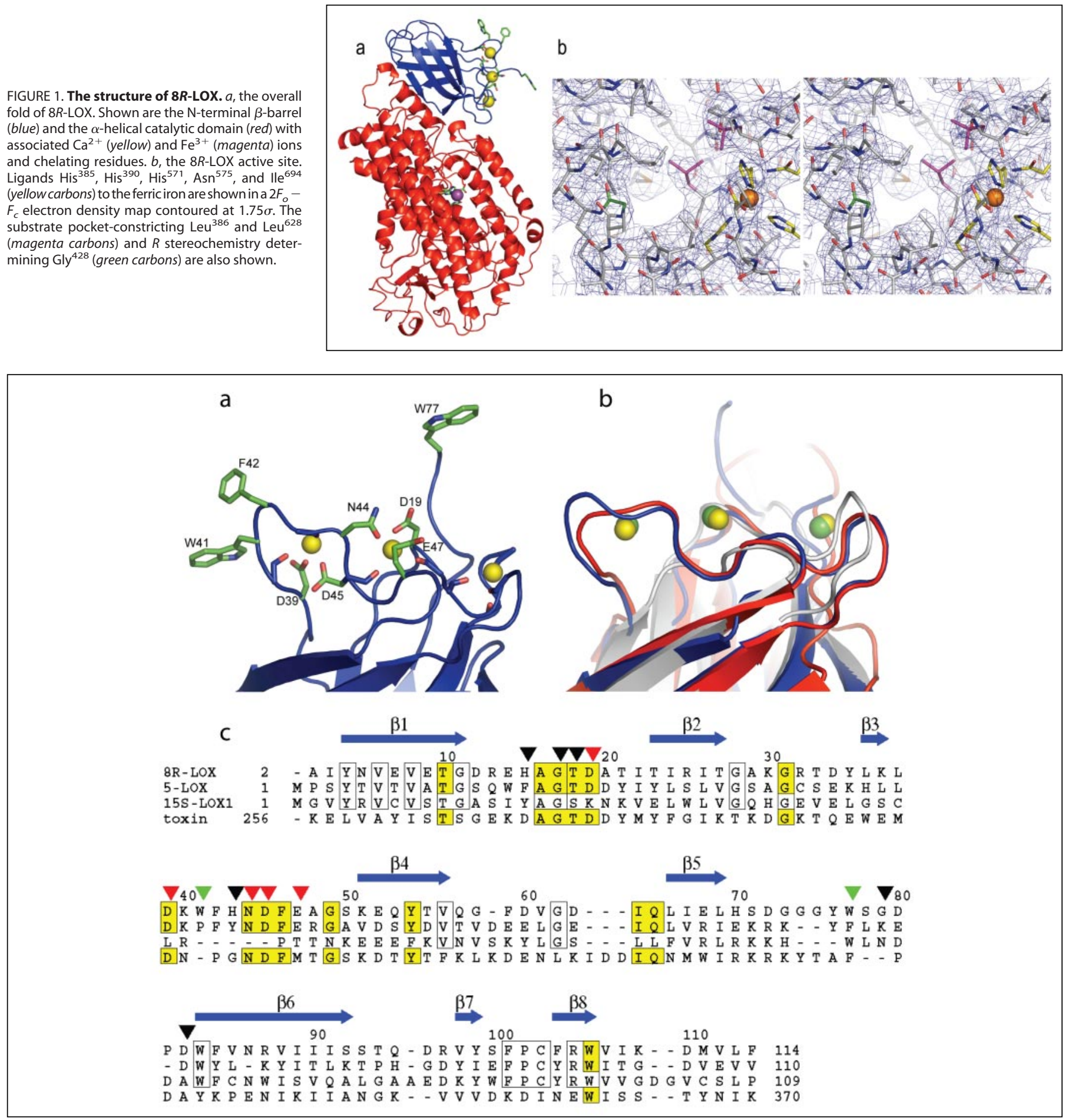

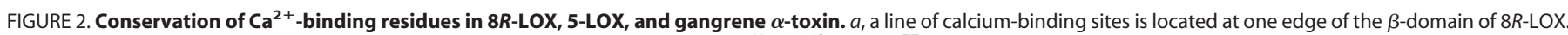

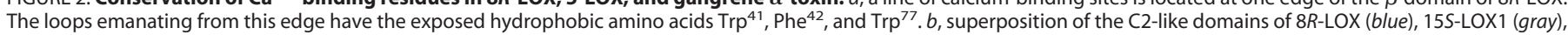

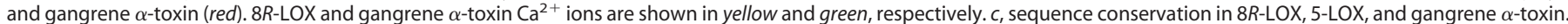

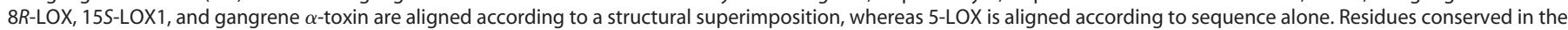

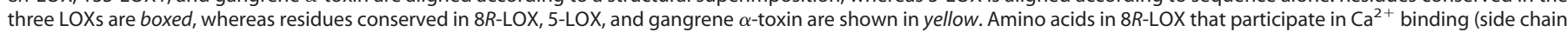
(red arrowheads) and main chain (black arrowheads) or membrane insertion (green arrowheads) are indicated.

$8 R$-LOX is shaped roughly like a cylinder $\sim 100 \AA$ in length and $60 \AA$ in diameter. The molecule is composed of two domains: an $\mathrm{N}$-terminal C2-like eight-stranded antiparallel $\beta$-barrel domain (residues 2-114) that constitutes the first third of the cylinder and a larger catalytic domain (residues 115-694) that contains $\sim 23$ helices and a threestranded antiparallel $\beta$-sheet (Fig. 1). The catalytic iron is centrally located in the $\alpha$-helical domain, but slightly displaced toward one surface. The iron is chelated by $\mathrm{His}^{385}, \mathrm{His}^{390}$, $\mathrm{His}^{571}, \mathrm{Asn}^{575}$, and $\mathrm{Ile}^{694}$ via its $\mathrm{C}$ terminus. A large elongated pocket passes by the iron, but this pocket does not have clear access to the surface. The shortest route to gain access to the cavity is by the movement of $\mathrm{Tyr}^{179}$ on a helix that forms the outermost wall of the internal cavity. 
FIGURE 3. Quenching of $8 \boldsymbol{R}$-LOX Trp fluorescence by dansyl-phosphatidylcholine/phosphatidylserine liposomes with the addition of $\mathrm{Ca}^{2+}$. The plot of $\Delta F_{ \pm \text {calcium }} / F_{- \text {calcium }} v e r s u s$ dansyl-liposome concentration could be fitted by nonlinear regression analysis to yield a hyperbolic binding curve with a $K_{d}$ of $11.4 \pm 2.7 \mu \mathrm{m}$. The protein concentration was $0.4 \mu \mathrm{m}$ in $50 \mathrm{~mm}$ Tris $(\mathrm{pH}$ 7.5), $500 \mathrm{~mm} \mathrm{NaCl}, 2$ mм EDTA. Emission spectra were recorded (excitation wavelength of $295 \mathrm{~nm}$ ), and calcium was added to a concentration of $4 \mathrm{~mm}$ prior to rescanning. $F_{330}$ is the fluorescence at $330 \mathrm{~nm}$.

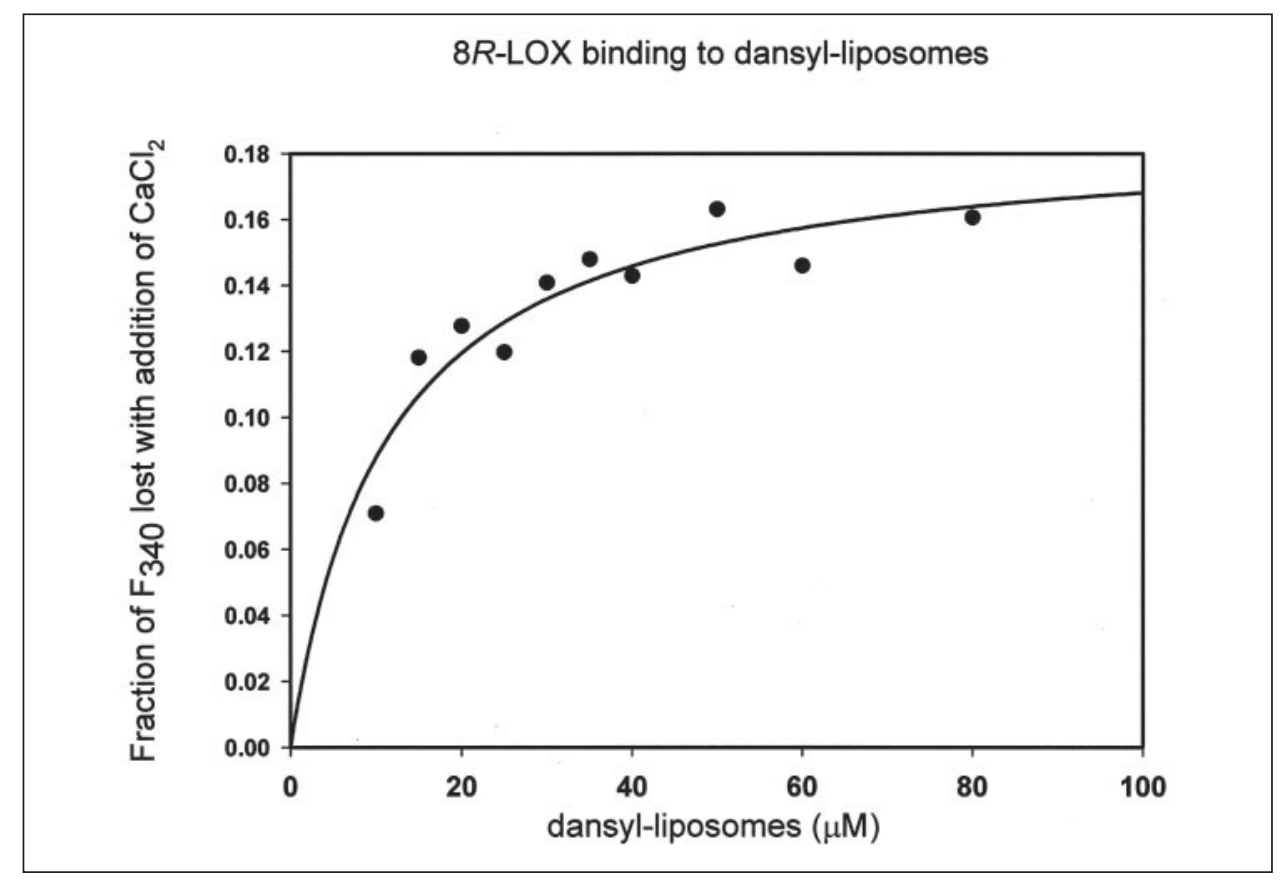

The C2-like $\beta$-Barrel Domain Contains Calcium Sites and Exposed Tryptophans-Structural features in the $\mathrm{N}$-terminal $\beta$-barrel domain unique to the $8 R$-LOX structure were apparent in the initial electron density map. The presence of $5 \sigma$ electron density peaks, an absolute requirement for calcium in the crystallization, and the presence and configuration of calcium-chelating amino acids led to the identification of three calcium-binding sites. $\mathrm{Ca}^{2+}$ is a crucial regulator of human leukocyte 5-LOX activation (6) and its nuclear membrane localization (23); but no crystal structure is available for this key enzyme, and these calcium and membrane binding features are lacking in the previously solved 15S-LOX1 structure.

If the $\mathrm{N}$-terminal domain is viewed as two layers of $\beta$-sheet, three interwoven calcium-binding sites formed by five side chains (site 1: $\mathrm{Asp}^{39}$ and $\mathrm{Asp}^{45}$; and site 2: $\mathrm{Asp}^{19}, \mathrm{Asn}^{44}$, and $\mathrm{Glu}^{47}$ ) and eight carbonyl groups (Fig. 2) are located at one edge of the structure. The three calcium-binding sites, which form a ridge between the two layers of sheet, seem to brace the structure by stabilizing loops that emanate from strands $\beta 3$ and $\beta 5$. These loops, which traverse the ridge from one layer to the other, contain surface-exposed hydrophobic amino acids ( $\beta 3$ : $\operatorname{Trp}^{41}$ and $\mathrm{Phe}^{42}$; and $\beta 5$ : $\operatorname{Trp}^{77}$ ). The loop that contains $\operatorname{Trp}^{77}$ is not well defined in the electron density map, whereas the loop that contains $\operatorname{Trp}^{41}$ is found nestled in a hydrophobic pocket of a symmetry mate in the context of the crystal packing. These tryptophans lie at either end of the line of the three $\mathrm{Ca}^{2+}$ ions, with one near the "top" layer of the $\beta$-structure and the other at the "bottom" layer. As is evident from the sequence alignment provided in Fig. 2, as well as the superposition of the $\beta$-barrel domains of $8 R$-LOX and 15S-LOX1 (r.m.s.d. of $1.506 \AA$ for 97 of 109 C- $\alpha$ atoms) (8) and gangrene $\alpha$-toxin (r.m.s.d. of $1.460 \AA$ for 93 of $113 \mathrm{C}$ - $\alpha$ atoms) (24), the calcium-binding residues found in $8 R$-LOX correspond to those observed in the gangrene $\alpha$-toxin domain and proposed for human 5-LOX (5), indicating the direct relevance of the $8 R$-LOX structure to elucidating aspects of calcium activation. Notably, the calcium-binding ridge and putative membrane insertion loops are on the same side of the molecule as the likely entrance to the cavity that holds the catalytic iron. Thus, a two-pronged grip of the $\beta$-barrel on the membrane could serve to orient the catalytic domain to facilitate the acquisition of substrate from the membrane phase.
Membrane Binding Affinity - The striking similarity of the structures of the $\beta$-barrel domains of $8 R$-LOX and gangrene $\alpha$-toxin (24) in the presence of $\mathrm{Ca}^{2+}$ led us to investigate the $\mathrm{Ca}^{2+}$-dependent membrane binding of $8 R$-LOX. We employed a FRET assay in which tryptophan served as the donor and dansyl-phospholipids incorporated into liposomes served as the acceptor. Membrane binding was indicated by a reduction in Trp fluorescence $(330 \mathrm{~nm})$ accompanied by an increase in dansyl fluorescence $(517 \mathrm{~nm})$. A binding isotherm based on the FRET data was constructed to determine the affinity of $8 R$-LOX for liposomes. The plot of the fraction of Trp fluorescence quenched in the presence of $\mathrm{CaCl}_{2}$ as a function of dansyl-liposome concentration is shown in Fig. 3. The data could be fitted by nonlinear regression analysis to yield a hyperbolic binding curve with a value of $11.4 \pm 2.7 \mu \mathrm{M}$ for the $8 R$-LOX/ liposome dissociation constant. In control experiments in the absence of $\mathrm{Ca}^{2+}$, no quenching of Trp fluorescence by dansyl-liposomes was observed, an indication that, in the absence of $\mathrm{Ca}^{2+}, 8 R$-LOX does not bind membranes to an appreciable extent.

To evaluate the effects of the mutation of putative $\mathrm{Ca}^{2+}$-ligating amino acids on $\mathrm{Ca}^{2+}$-mediated membrane insertion, the increase in fluorescence at $517 \mathrm{~nm}$ upon the addition of $\mathrm{CaCl}_{2}$ to enzyme/dansylliposome mixtures was monitored (excitation wavelength of $295 \mathrm{~nm}$ ) for wild-type and mutant 8R-LOXs at liposome concentrations of 260 $\mu \mathrm{M}$. As shown in Fig. 4, a distinct increase in fluorescence at $517 \mathrm{~nm}$ was observed for the wild-type enzyme with the addition of calcium to the assay. Mutants in which a single $\mathrm{Ca}^{2+}$-binding residue had been replaced with Ala (D39A and E47A) exhibited diminished FRET properties, consistent with a role for calcium in membrane binding. A double mutant (D39A/E47A) with calcium-binding residues from two of the three sites mutated exhibited no FRET signal at all.

As mentioned above, the calcium-binding residues appear to anchor the $\beta$-barrel loops that contain $\operatorname{Trp}^{41}$ and $\operatorname{Trp}^{77}$ (Fig. 2). These two loops emanate from the same edge of the $\beta$-sheet stack, one on each of the top and bottom layers. Thus, the loops could simultaneously insert to mediate a two-pronged attachment to the membrane. In the context of the crystal packing, the loop that contains $\operatorname{Trp}^{41}$ is found nestled in a hydrophobic cavity in a neighboring symmetry mate, whereas the loop that contains $\operatorname{Trp}^{77}$ is not fully ordered. W41A exhibited $<2 \%$ of the increase 


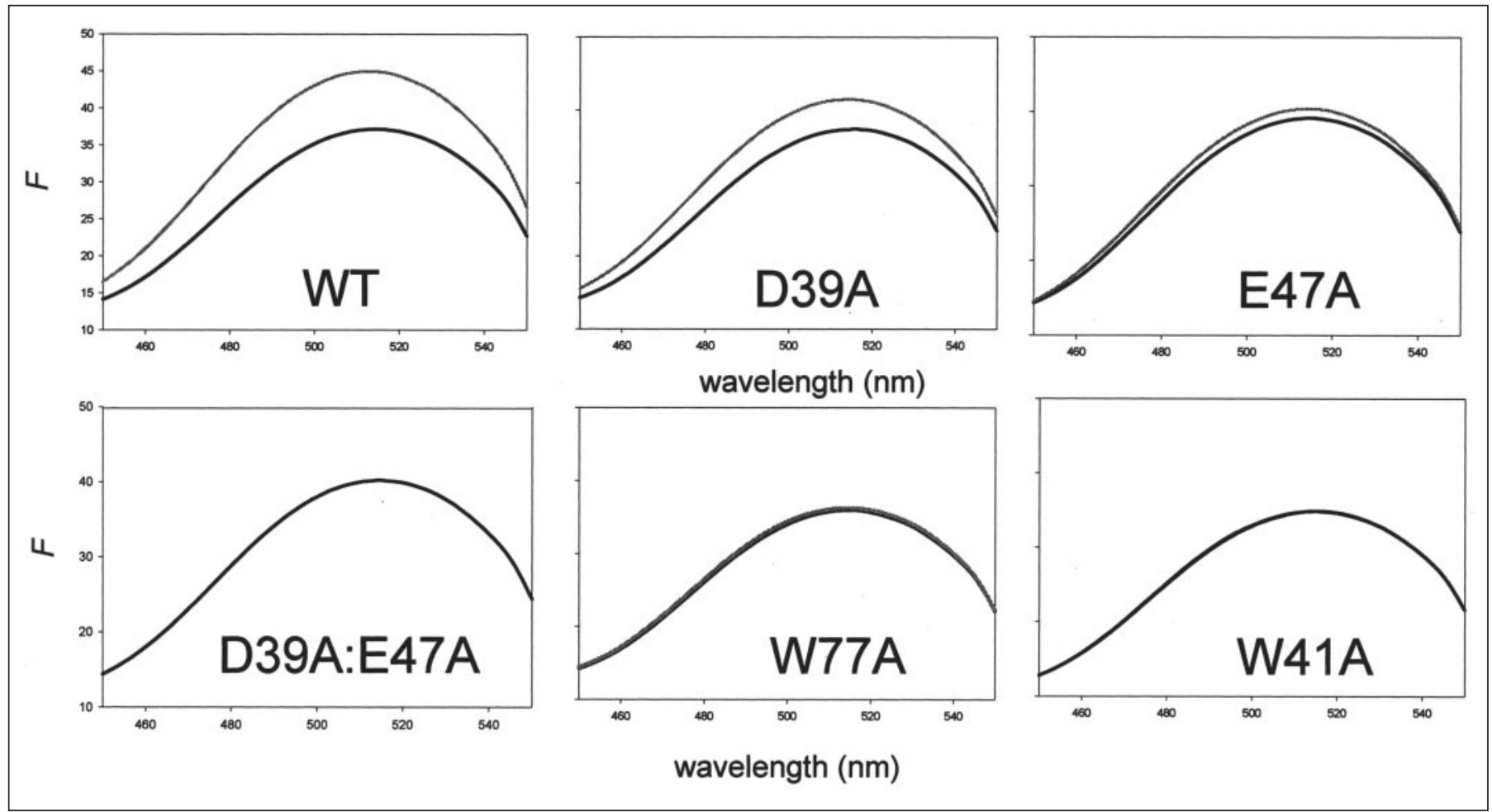

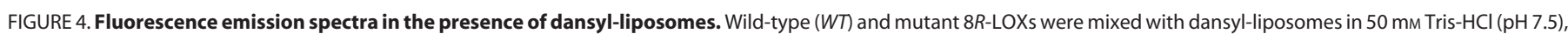
$500 \mathrm{~mm} \mathrm{NaCl}$, and $4 \mathrm{~mm}$ EDTA and scanned in the absence (black traces) and presence (gray traces) of $6 \mathrm{~mm} \mathrm{CaCl}_{2}$ (excitation wavelength of $295 \mathrm{~nm}$ ).

in fluorescence at $517 \mathrm{~nm}$ upon the addition of $\mathrm{CaCl}_{2}$. Similarly, W77A exhibited only $4 \%$ of the wild-type signal. The loss of signal is consistent with either the loss of membrane binding affinity and/or simply the loss of a source of donor fluorescence for the FRET signal. The fact that both mutations (W41A and W77A) individually led to a loss of signal suggests that both loops are important for membrane binding or the FRET signal.

Effect of Calcium on Enzyme Activity in the Presence of LiposomesGiven the effect of calcium on membrane binding and the fact that the substrate arachidonic acid partitions into the membrane phase, we asked whether impaired membrane binding might result in a reduction in enzyme activity in the presence of membranes. In a liposome-based assay, wild-type activity was clearly stimulated upon the addition of $\mathrm{Ca}^{2+}$. However, a plot of the change in absorbance at $235 \mathrm{~nm}$ versus time appears to suggest that the reaction reached completion at a concentration of $1 \mu \mathrm{M}$ substrate (Fig. $5 a$ ), whereas the concentration of arachidonic acid in the reaction mixture was $25 \mu \mathrm{M}$. However, not all substrate is available to the membrane-bound enzyme, as the solubility of arachidonic acid under similar conditions is appreciable (with a critical micellar concentration of $>50 \mu \mathrm{M}(25)$ ), and substrate in the aqueous phase is unavailable to the membrane-bound enzyme. Although arachidonic acid on the outer leaflet is accessible and rapidly consumed, subsequent turnovers depend on the re-equilibration of substrate between the aqueous and membrane phases.

We monitored the activity of $8 R$-LOX mutants in which the calciumbinding residues and putative membrane insertion residues were mutated. The results of these experiments support the FRET data, as mutations that abrogated binding of $8 R$-LOX to membranes also eliminated $\mathrm{Ca}^{2+}$ stimulation of enzyme activity in the presence of liposomes (Fig. 5). Specifically, the double mutant D39A/E47A, which did not induce an increase in dansyl fluorescence upon the addition of calcium, did not have enhanced activity in the presence of $\mathrm{Ca}^{2+}$. Similarly, the
D39A and E47A single mutants showed partial stimulation of activity by calcium and a reduced membrane-bound acceptor fluorescence emission in the FRET assay. The $\mathrm{Ca}^{2+}$ stimulation of the enzyme activity of the W41A mutant was also diminished. This observation supports a role for $\operatorname{Trp}^{41}$ in membrane insertion. However, this mutant showed intermediate stimulation of activity by $\mathrm{Ca}^{2+}$, and this observation may indicate that the enzyme could still weakly associate with the membrane. The results of the activity assay with the W77A mutant are not easily interpreted, as this form of the enzyme had reduced activity in a membrane-free assay, yet significantly enhanced activity in the presence of liposomes with and without $\mathrm{Ca}^{2+}$. These anomalous results, along with a low yield from the protein expression and purification protocol that proved to be successful with other forms of the prepared enzyme, suggest that this substitution may have destabilized the native conformation.

The Catalytic Domain - 8R-LOX has 30\% sequence identity to rabbit 15S-LOX1, for which a crystal structure has been reported (8). The structural determination of 15S-LOX1 required the presence of an aryl carboxylate competitive inhibitor (RS75091) (8). This compound was crucial in the preparation of diffraction quality crystals of the rabbit enzyme and further allowed for the identification of the substrate-binding site. However, the competitive inhibitor does not appear to be a true substrate mimic, as its carboxylate is positioned proximal to the nonheme iron, blocking approach to the open position in the coordination sphere of the metal. In contrast, the surface-exposed $\mathrm{Arg}^{403}$ is thought to tether the substrate by interaction with the arachidonic acid carboxylate group. Thus, a clear interpretation of conformational differences between the substrate cavities of the two enzymes is complex, as these differences may reflect not only specificity and product differences, but also the fact that one of the models is of an inhibited enzyme. Nevertheless, obvious differences can be described.

In Fig. $6 a$, the structures of the rabbit and coral LOXs are superimposed, with the models oriented such that the postulated site of entry to 

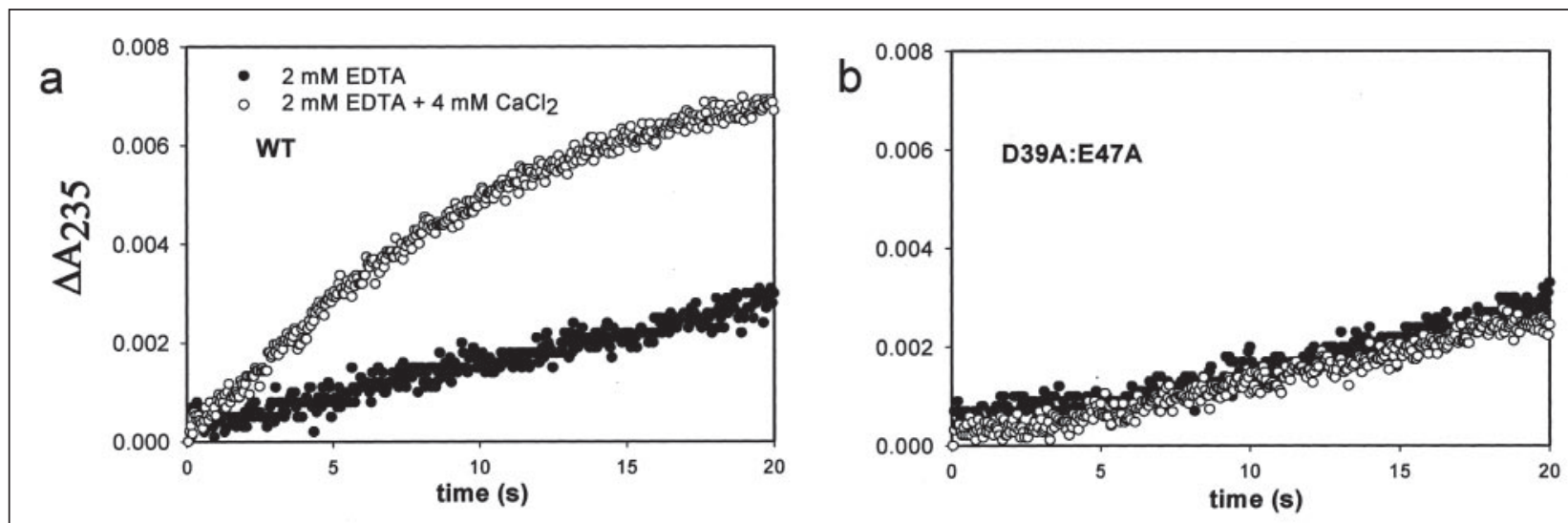

C

\begin{tabular}{|l|c|c|c|c|}
\hline Enzyme & \multicolumn{2}{|c|}{$\begin{array}{c}\text { Activity relative to wt-LOX } \\
\text { (\%) }\end{array}$} & Fold stimulation & $\begin{array}{c}\text { Enhancement of } \mathbf{F}_{\text {517 }} \text { relative to } \\
\text { wild type (\%) }\end{array}$ \\
& AA & AA-LUV & AA-LUV + $\mathbf{C a C l}_{\mathbf{2}}$ & Dansyl-LUV + $\mathbf{C a C l}_{\mathbf{2}}$ \\
wild-type & 100 & 100 & $4.0 \mathrm{X}$ & $100(3.2)$ \\
D39A & 118 & 100 & $2.2 \mathrm{X}$ & $15(5)$ \\
E47A & 65 & 115 & $2.8 \mathrm{X}$ & $40(5)$ \\
D39A:E47A & 106 & 108 & $0.9 \mathrm{X}$ & Not detected \\
W77A & 44 & 315 & $4.1 \mathrm{X}$ & $4(1)$ \\
W41A & 140 & 123 & $2.7 \mathrm{X}$ & $<2$ \\
\hline
\end{tabular}

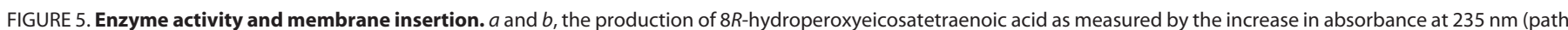

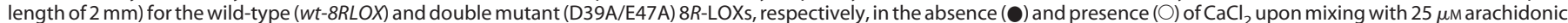

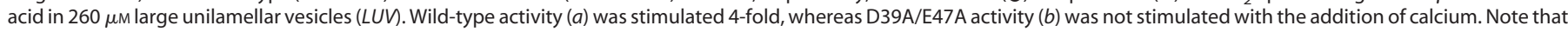

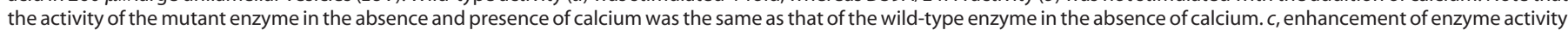

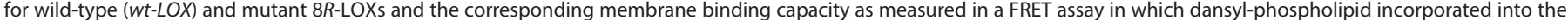
membrane vesicles was an acceptor for $\operatorname{Trp}$ fluorescence. $A A$, arachidonic acid; $F_{517}$, fluorescence at $517 \mathrm{~nm}$

the active site is from above (r.m.s.d. of $1.54 \AA$ for 576 of $662 \mathrm{C}$ - $\alpha$ atoms). The placement of the elements of secondary structure is strikingly conserved at the "lower" half of the catalytic domain, where the long $\alpha$-helices clearly overlay. Two of these helices contribute four of the ironligating side chains $\left(\mathrm{His}^{385}, \mathrm{His}^{390}, \mathrm{His}^{571}\right.$, and $\left.\mathrm{Asn}^{575}\right)$. At the entrance side of the catalytic domain, there is more deviation in the positions of the shorter helices. The most notable difference here is a helical segment in $8 R$-LOX that covers access to the catalytic iron. The corresponding region is not ordered in the rabbit structure, and inspection of the $15 S$-LOX1 crystal packing context indicates that a helical region here is not compatible with the crystal packing. This observation further complicates our interpretation of structural differences, as one cannot rule out the possibility that crystal packing has selected for the observed rabbit LOX conformation.

The substrate-binding pocket of $8 R$-LOX is illustrated in Fig. $6 b$. In this rendering of a slab through the active site, internal voids appear as clouds outlining two well resolved inner cavities (for the sake of discussion, denoted Cavity A and Cavity B); the catalytic iron is located in the larger of these cavities (Cavity A). Both cavities are concealed from bulk solvent. We have modeled in the 20 -carbon substrate such that $\mathrm{C}-10$ is positioned for $\mathrm{H}^{+}$abstraction by $\mathrm{Fe}^{3+}$ and $\mathrm{C}-8$ is positioned for attack by $\mathrm{O}_{2}$ in a pouch flanked by $\mathrm{Gly}^{428}$, the position that determines product stereochemistry (16). It is remarkable that, in comparison with
$S$-LOX(s), the structural consequence of the presence of the Gly (versus Ala) on helix 13 (the second helix following the long helix contributing $\mathrm{His}^{385}$ and $\mathrm{His}^{390}$ to the $\mathrm{Fe}^{3+}$ coordination sphere) appears to be primarily one of freeing up space. A significant realignment of this helix, which forms a substantial fraction of the active site, is not observed.

Two conformational changes are required for our proposed mode of substrate binding. Movement of $\mathrm{Tyr}^{179}$ into a side pocket of Cavity A permits association of the substrate carboxylate with the surface-exposed $\mathrm{Arg}^{183}$. In the observed conformation, there is no access to the catalytic iron, and the repositioning of $\mathrm{Tyr}^{179}$ is also necessary to facilitate entry into the active site. Deeper in the pocket, a constriction between Cavities A and B blocks access to the hydrocarbon tail of arachidonic acid, but a rotamer change in $\mathrm{Leu}^{386}$ and a slight shift of Leu ${ }^{628}$ would open up the constriction. These conformational changes we propose can be adapted with minimal consequences to the protein structure, as they are largely confined to rotamer changes that fill existing pockets. In the structure of $15 S$-LOX1 with inhibitor, the region that corresponds to the helix that blocks solvent access in $8 R$-LOX is disordered. (The carboxylate of arachidonic acid has access to the surfaceexposed $\mathrm{Arg}^{403}$ of that enzyme.) Our $8 R$-LOX structure suggests that disordering of the helix that contains $\mathrm{Tyr}^{189}$ and $\mathrm{Arg}^{183}$ is not necessary to gain entry to the active site. 


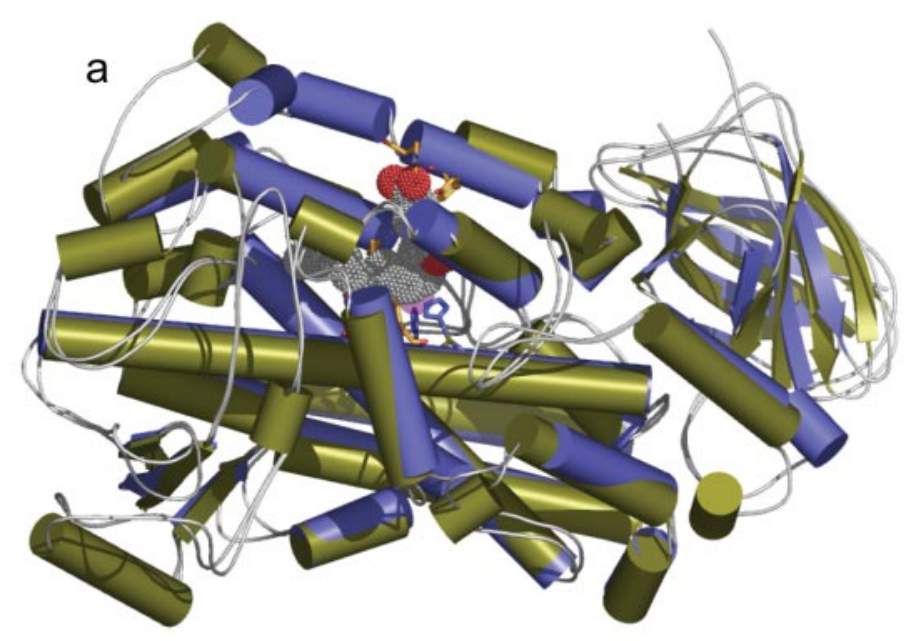

b

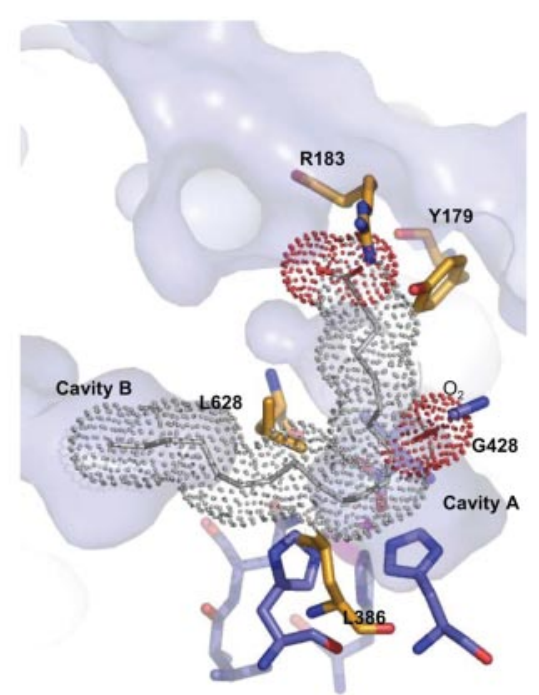

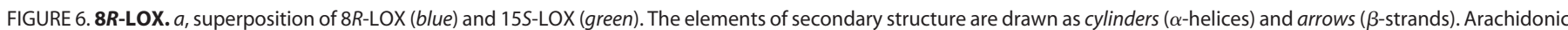

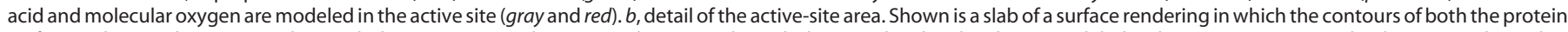

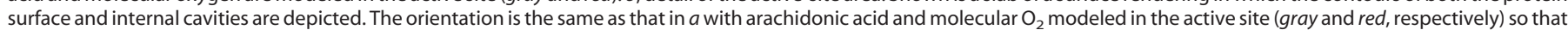

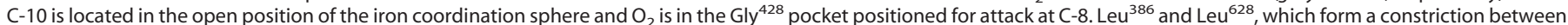

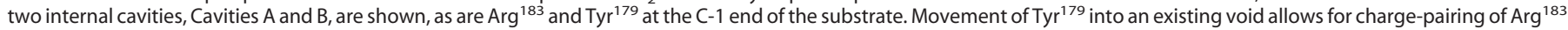

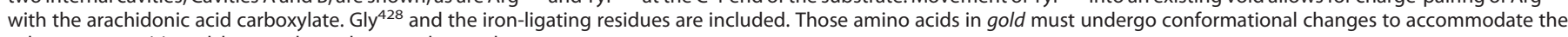
substrate as positioned, but are drawn here as observed.

\section{DISCUSSION}

The binding of human 5-LOX to the nuclear membrane is regulated by $\mathrm{Ca}^{2+}$. Based on the sequence identity to the membrane-targeting domain of gangrene $\alpha$-toxin and site-directed mutagenesis experiments, $\mathrm{Ca}^{2+}$-binding amino acids have been identified in its $\mathrm{N}$-terminal domain. Calcium has also been shown to promote the binding of $15 \mathrm{~S}$ LOX1 to membranes; however, this interaction does not appear to require specific $\mathrm{Ca}^{2+}$-binding amino acids (7). The structure of $8 R$-LOX reveals clearly defined calcium-binding sites in its C2-like domain, and the chelating residues are conserved in both 5-LOX and gangrene $\alpha$-toxin. Furthermore, we have presented data consistent with a role for $\mathrm{Ca}^{2+}$ in membrane binding and demonstrated that enzyme activity is enhanced by $\mathrm{Ca}^{2+}$ in the presence of liposomes. $\mathrm{Ca}^{2+}$ stimulation of enzyme activity is likely a result of targeting the enzyme to the membrane phase, where higher concentrations of substrate can be found. In its marine environment, $P$. homomalla is osmotically equilibrated with sea water, which contains $5-10 \mathrm{mM} \mathrm{CaCl}_{2}$. However, the free cytosolic calcium concentration, like that in typical plant and animal cells, might be as low as nanomolar. It is unclear whether $8 R$-LOX translocates to the membrane during transient calcium stimulation or whether the bound calcium ions serve as part of a static structural element that promotes $8 R$-LOX sequestration at the membrane.

Our data suggest that $\mathrm{Ca}^{2+}$ binding stimulates enzyme activity by targeting $8 R$-LOX to the membrane. The $\mathrm{Ca}^{2+}$-binding ridge with membrane insertion loops that we have described is on the same side of the cylindrically shaped LOX molecule as the entrance to the binding site and thus provides a means to effectively tether the enzyme at the bilayer and to optimize substrate acquisition. However, we cannot exclude calcium-induced or membrane binding-induced conformational changes that might also impact enzyme activity. Although the correlation of the FRET data with the enzyme activity data suggests that membrane targeting is a significant part of the activation mechanism, Boutaud and Brash (26) previously reported an increase in $8 R$-LOX activity in the presence of calcium in a membrane-free assay. Thus, the question remains whether the $\mathrm{C} 2$-like domain may be a regulatory domain as well as a membrane-targeting domain. Preparations of catalytic domain-only LOXs have altered catalytic activity. Although the proteolytically cleaved catalytic domain of soybean LOX L1 has higher activity and lower stability (27), the truncated version of 15S-LOX1 has reduced activity (28). Both observations suggest that there is communication between the domains, and all $\mathrm{x}$-ray structures available have revealed extensive contact interfaces that would allow for interdomain communication. Significantly, calcium binding to the C2-like domain of gangrene $\alpha$-toxin results in the ordering of a short segment of the phospholipase domain $(4,24)$, although it is unclear whether $\mathrm{Ca}^{2+}$ impacts catalytic activity per se or simply enzyme localization.

As mentioned above, LOX N-terminal C2-like domains have been implicated in membrane binding, and $\mathrm{C} 2$ domains are often found in proteins that participate in $\mathrm{Ca}^{2+}$-regulated membrane insertion $(3,29$, 30). The sequence identity observed between 5 - LOX and the C2-like domain of gangrene $\alpha$-toxin, a membrane-binding phospholipase, led investigators to model the $\mathrm{N}$-terminal region of 5-LOX on the $\alpha$-toxin template and demonstrate that putative surface-exposed tryptophan residues in 5-LOX $\beta$-barrel loops are required for $\mathrm{Ca}^{2+}$-regulated membrane binding (5). Site-detected mutagenesis studies indicate that calcium is bound in part by a set of acidic residues found at the base of the $\beta$-barrel loops (6). Moreover, $\mathrm{Ca}^{2+}$ is a key regulator in 5-LOX activation (6) and nuclear membrane localization (23). However, no crystal structure is available for 5-LOX, and these calcium and membrane binding features are lacking in 15S-LOX1.

The affinity of $\mathrm{Ca}^{2+}$-loaded $\mathrm{C} 2$ domains for membranes varies among superfamily members; for example, the membrane binding dissociation constants for $\mathrm{C} 2$ domains from synaptotagmin I, protein kinase $C \beta$, and phospholipase $A_{2}$ range from 3.4 to $24 \mu \mathrm{M}$ (31). Although the LOX membrane-binding domain is a variation on a $\mathrm{C} 2$ theme, it is a distinctly different module not only in its $\beta$-barrel topology, but also in terms of the $\mathrm{Ca}^{2+}$ stabilization of membrane-binding loops. Where the LOX C2-like domain has a line of $\mathrm{Ca}^{2+}$ ions flanked by membrane insertion loops, the ions bound by typical $\mathrm{C} 2$ domains cluster in "jaws" formed by loops that emanate from the barrel. Although C2 


\section{Calcium Regulation of 8R-Lipoxygenase}

domain calcium sensors are found in phospholipases, kinases, and membrane trafficking proteins, homologs of the C2-like domain appear to be largely restricted to LOXs and bacterial phospholipases (e.g. gangrene $\alpha$-toxin). Furthermore, not all of the homologs have the $\mathrm{Ca}^{2+}$ binding amino acids common to 5-LOX, coral LOX, and $\alpha$-toxin.

Tatulian and co-workers (32) have proposed a model for the interaction of 5-LOX with the membrane. Their spectroscopic data suggest that membrane fluidity is necessary to allow insertion of $\operatorname{Trp}^{75}$ of the C2-like domain ( $\operatorname{Trp}^{77}$ in 8R-LOX) as deep as 8-9 $\AA$ from the center of the bilayer and that $\sim 9600 \AA^{2}$ of LOX make contact with the membrane. Our data for $8 R$-LOX are consistent with such a model, as the loop that includes $\operatorname{Trp}^{77}$ is fully extended and mobile, and mutation of this amino acid resulted in a loss of membrane-binding FRET signal. Pande et al. (32) have reported a $K_{d}$ of $0.78 \mu \mathrm{M}$ for binding of 5-LOX to 1-palmitoyl-2-oleoyl-sn-glycero-3-phosphocholine vesicles, a value significantly different from that for binding of the isolated C2-like domain to vesicles of similar composition (0.6 nM) (5). With mixed phosphatidylcholine/phosphatidylserine vesicles, we obtained a $K_{d}$ of $11 \mu \mathrm{M}$, a value consistent with some aspects of the membrane-binding mechanisms common to $8 R$-LOX and 5-LOX.

The structure of $8 R$-LOX is the first for a LOX that produces a fatty acid hydroperoxide $R$-stereoisomer. It is well established that the first step of the reaction with substrate involves the stereospecific hydrogen abstraction by the activated $\mathrm{Fe}^{3+} \mathrm{OH}$, from position 10D in the case of $8 R$-LOX (12), to produce a pentadiene free radical intermediate. This $\mathrm{C}-10$ hydrogen abstraction potentially activates the fatty acid for oxygenation at either $\mathrm{C}-8$ or $\mathrm{C}-12$; and as oxygenation is known to occur on the opposite face of the activated substrate, the product is either the $8 R$ or $12 S$-stereoisomer. As noted previously, an Ala (or Ser) is conserved in the binding site of $S$-LOXs, and this is replaced with Gly in 8R-LOX and other LOXs with $R$ specificity; site-directed mutagenesis of $\mathrm{Gly}^{428}$ $\left(\right.$ Gly $^{427}$ in the numbering scheme of Coffa and Brash (16)) to Ala in $8 R$-LOX yields $12 S$-product, whereas the corresponding Ala-to-Gly mutation in mouse $12 S$-LOX produces $8 R$-product (16). One of the implications of this previous study relates to the head-to-tail orientation of substrate binding in the active site, and we used the prediction of tail-first binding for $8 R$-LOX to model arachidonic acid in the substratebinding cavity (Fig. $6 b$ ). The contours of the pocket support the idea that one molecule of $\mathrm{O}_{2}$ can be accommodated in a pouch between the catalytic iron and the protein surface, thus facilitating $8 R$ oxygenation. Less clear at our current structural resolution of $3.2 \AA$ is what prevents reaction with molecular oxygen at $\mathrm{C}-12$ of the substrate in the deeper recesses of the pocket. The $8 R$-LOX G428A mutant yields the $12 S$ product, yet its activity is $\sim 20 \%$ of that of the wild-type enzyme (16). A possible interpretation of this result is that the space available to $\mathrm{O}_{2}$ at position 12 is suboptimal. In future studies, we will crystallize the $8 R$-LOX G428A mutant and examine this issue directly. In both the $8 R$-LOX and 15S-LOX1 structures, there appears to be room in the cavity for both substrate and $\mathrm{O}_{2}$ beyond the catalytic iron, where oxygenation at C-12 (or C-15 in 15-LOX) would occur. However, the presence of a constriction that must open to accommodate the tail of arachidonic acid as described above for $8 R$-LOX and the fact that interpretations of cavity contour differences in the rabbit and coral enzymes are complicated by differences in crystallization conditions (the former in the presence of inhibitor and the latter in the absence of substrate) make it difficult to position the tail or oxygen with certainty beyond the catalytic iron.
What is clear from the current structure determination and comparison with $15 S$-LOX1 is that the presence of the critical glycine $\left(\mathrm{Gly}^{428}\right)$ of $8 R$-LOX has minor effects on the structure, other than creation of a small side pocket that can potentially accommodate $\mathrm{O}_{2}$ (Fig. 6). For $8 R$-LOX, with substrate-bound hydrocarbon tail first, Gly ${ }^{428}$ leaves space for an oxygen pocket close to C-8, and it appears that this, together with shielding of the other reactive positions by the protein, leads to the specific production of the $8 R$-hydroperoxide product.

Acknowledgment-We thank William Boeglin for assistance in construct design.

\section{REFERENCES}

1. Brash, A. R. (1999) J. Biol. Chem. 274, 23679-23682

2. Kuhn, H., and Thiele, B. J. (1999) FEBS Lett. 449, 7-11

3. Rizo, J., and Sudhof, T. C. (1998) J. Biol. Chem. 273, 15879-15882

4. Naylor, C. E., Eaton, J. T., Howells, A., Justin, N., Moss, D. S., Titball, R. W., and Basak, A. K. (1998) Nat. Struct. Biol. 5, 738-746

5. Kulkarni, S., Das, S., Funk, C. D., Murray, D., and Cho, W. (2002) J. Biol. Chem. 277, 13167-13174

6. Hammarberg, T., Provost, P., Persson, B., and Radmark, O. (2000) J. Biol. Chem. 275, 38787-38793

7. Walther, M., Wiesner, R., and Kuhn, H. (2004) J. Biol. Chem. 279, 3717-3725

8. Gillmor, S. A., Villasenor, A., Fletterick, R., Sigal, E., and Browner, M. F. (1997) Nat. Struct. Biol. 4, 1003-1009

9. Hornung, E., Rosahl, S., Kuhn, H., and Feussner, I. (2000) Biochem. Soc. Trans. 28, $825-826$

10. Jisaka, M., Kim, R. B., Boeglin, W. E., and Brash, A. R. (2000) J. Biol. Chem. 275, 1287-1293

11. Skrzypczak-Jankun, E., Bross, R. A., Carroll, R. T., Dunham, W. R., and Funk, M. O., Jr. (2001) J. Am. Chem. Soc. 123, 10814-10820

12. Hughes, M. A., and Brash, A. R. (1991) Biochim. Biophys. Acta 1081, 347-354

13. Boyington, J. C., Gaffney, B. J., and Amzel, L. M. (1990) J. Biol. Chem. 265, 12771-12773

14. Minor, W., Steczko, J., Stec, B., Otwinowski, Z., Bolin, J. T., Walter, R., and Axelrod, B. (1996) Biochemistry 35, 10687-10701

15. Skrzypczak-Jankun, E., Amzel, L. M., Kroa, B. A., and Funk, M. O., Jr. (1997) Proteins $29,15-31$

16. Coffa, G., and Brash, A. R. (2004) Proc. Natl. Acad. Sci. U. S. A. 101, 15579-15584

17. Koljak, R., Boutaud, O., Shieh, B. H., Samel, N., and Brash, A. R. (1997) Science 277, 1994-1996

18. Zhang, Y. Y., Hamberg, M., Radmark, O., and Samuelsson, B. (1994) Anal. Biochem. 220, $28-35$

19. Otwinowski, Z., and Minor, W. (1997) Methods Enzymol. 276, 307-326

20. Collaborative Computational Project (1994) Acta Crystallogr. Sect. D Biol. Crystallogr. 50, 760-763

21. Jones, T. A., Zou, J. Y., Cowan, S. W., and Kjeldgaard, M. (1991) Acta Crystallogr. Sect. A 47, 110-119

22. Adams, P. D., Pannu, N. S., Read, R. J., and Brunger, A. T. (1997) Proc. Natl. Acad. Sci. U. S. A. 94, 5018-5023

23. Chen, X. S., and Funk, C. D. (2001) J. Biol. Chem. 276, 811-818

24. Naylor, C. E., Jepson, M., Crane, D. T., Titball, R. W., Miller, J., Basak, A. K., and Bolgiano, B. (1999) J. Mol. Biol. 294, 757-770

25. Glick, J., Santoyo, G., and Casey, P. J. (1996) J. Biol. Chem. 271, 2949-2954

26. Boutaud, O., and Brash, A. R. (1999) J. Biol. Chem. 274, 33764-33770

27. Di Venere, A., Salucci, M. L., van Zadelhoff, G., Veldink, G., Mei, G., Rosato, N., Finazzi-Agro, A., and Maccarrone, M. (2003) J. Biol. Chem. 278, 18281-18288

28. Walther, M., Anton, M., Wiedmann, M., Fletterick, R., and Kuhn, H. (2002) J. Biol. Chem. 277, 27360-27366

29. Perisic, O., Paterson, H. F., Mosedale, G., Lara-Gonzalez, S., and Williams, R. L. (1999) J. Biol. Chem. 274, 14979-14987

30. Chapman, E. R., and Davis, A. F. (1998) J. Biol. Chem. 273, 13995-14001

31. Nalefski, E. A., Wisner, M. A., Chen, J. Z., Sprang, S. R., Fukuda, M., Mikoshiba, K., and Falke, J. J. (2001) Biochemistry 40, 3089-3100

32. Pande, A. H., Qin, S., and Tatulian, S. A. (2005) Biophys. J. 88, 4084-4094 